\title{
SÍNDROME DE BURNOUT EM PROFESSORES DAS ESCOLAS PÚBLICAS DO MUNICÍPIO DE BUENÓPOLIS, MG
}

\author{
BURNOUT SYNDROME IN TEACHERS OF PUBLIC SCHOOLS IN BUENÓPOLIS, MG, BRAZIL
}

\author{
Valéria Alaide de Araújo ${ }^{\mathrm{a}^{*}}$, Jéssica Magalhães Freire ${ }^{\mathrm{b}^{*}}$, Marcos Vinícius Macedo de Oliveira年** \\ avaleria--vivo@hotmail.com, bmagalhaesjessica5@gmail.com, cmvmoliv@gmail.com \\ *Faculdades Unidas do Norte de Minas - Montes Claros (MG), Brasil \\ **Faculdades Unidas do Norte Minas e Faculdades Integradas Pitágoras de Montes Claros - Montes Claros (MG), Brasil
}

Data de recebimento do artigo: 30/11/2016

Data de aceite do artigo: 15/02/2017

\section{RESUMO}

Introdução: A síndrome de burnout, ou síndrome do esgotamento profissional, está associada ao trabalho, ambiente no qual podem ocorrer atividades que causam desajustamentos psicofísicos nos profissionais. Sua maior incidência está entre os trabalhadores que têm relaçôes diretas com pessoas, como é o caso dos professores, que convivem diariamente com fatores que colaboram para desgastes mentais e físicos. Objetivo: Analisar a ocorrência da síndrome de burnout entre professores do município de Buenópolis (MG), verificando possíveis associaçôes com as características sociodemográficas e laborais desses profissionais. Materiais e métodos: Trata-se de um estudo analítico, transversal e quantitativo em uma amostra de 71 professores. Utilizou-se instrumentos de pesquisa como formulários socioeconômicos, laborais e o MBI (Maslach Burnout Inventory) que identificou características dos investigados e a ocorrência da síndrome de burnout. Resultados: Verificou-se que $52,1 \%$ dos professores apresentam a síndrome de burnout, cuja maior ocorrência foi observada em indivíduos que se sentem pouco valorizados $(\mathrm{p}=0,001)$, com pouca autonomia $(\mathrm{p}=0,000)$, que não gostam do trabalho $(\mathrm{p}=0,014)$, e que já pensaram em desistir da profissão $(\mathrm{p}=0,000)$. Conclusáo: $\mathrm{O}$ trabalho docente é uma profissão que pode gerar grande estresse, e com a análise deste estudo evidencia-se que mais da metade dos professores analisados apresenta a síndrome de burnout. Existe a necessidade de melhorar o ambiente organizacional tendo em mente os fatores relacionados à doença, visto que essa gera agravos à saúde e ao desempenho do professor, prejudicando também o sistema educacional. Sugere-se, assim, como forma de melhoria, o acompanhamento psicopedagógico como caráter profilático.

Palavras-chave: Burnout, esgotamento profissional; educação; trabalho.

\section{ABSTRACT}

Introduction: The burnout syndrome is associated with work. In this environment, there may be activities that cause psychophysical maladjustment in workers. Its incidence is higher among professionals who have direct relationships with people, such as teachers, who live daily with factors that contribute to mental and physical fatigue. Objective: To analyze the occurrence of burnout syndrome among teachers in the city of Buenópolis, Minas Gerais, Brazil, checking if there is any association with sociodemographic characteristics and work of these professionals. Materials and methods: This is an analytical, transversal and quantitative study on a sample of 71 teachers. Some research tools were used, such as socioeconomic forms, labor and MBI (Maslach Burnout Inventory) that identified characteristics and the occurrence of burnout syndrome. Results: It was found that $52.1 \%$ of the teachers have burnout syndrome, and a higher incidence of this was observed among individuals who feel undervalued ( $\mathrm{p}=0.001)$, with little autonomy $(\mathrm{p}=0.000)$, who do not like the job $(\mathrm{p}=0.014)$, and have thought of giving up the profession $(\mathrm{p}=0.000)$. Conclusion: The teaching profession can generate great stress, and the analysis of this study shows that more than half of the teachers analyzed presented burnout syndrome. There is a need for improvement in the organizational environment keeping in mind the factors related to the disease, since it causes health problems and affects teacher performance, hampering the educational system. We suggest, as a way to improve conditions, the psycho-pedagogical supervision as prophylactic treatment.

Keywords: Burnout; professional fatigue; education; job. 


\section{Introdução}

Maior exigência profissional surgiu com as mudanças ocorridas na sociedade nos últimos anos. Assim, para um bom profissional destacar-se em suas qualidades, é necessário saber agir em situaçóes imprevistas ${ }^{1}$. A grande competição por trabalho exige profissionais mais bem qualificados para ocupar as vagas no mercado, gerando nestes um grande estresse físico e mental ${ }^{2}$, fazendo que o prazeroso, fonte de liberdade e autorrealização, torne-se um fator estressor, levando à evolução de doenças ${ }^{3}$.

A saúde do trabalhador vem sendo foco de muitos estudos, pois grande parte das profissóes pode gerar um aumento de estresse e desgaste na vida pessoal e profissional e, dessa maneira, por muitas vezes, agravos à saúde decorrentes desse esgotamento podem ser irreversíveis ${ }^{4}$. O desgaste gerado na atividade ocupacional é conhecido como síndrome de burnout e está diretamente ligado ao ambiente de trabalho, onde os profissionais demonstram alteraçóes físicas e psicológicas decorrentes dos desajustamentos às atividades prestadas 5 . A síndrome pode ser evidenciada a partir de três características gerais: exaustão emocional, despersonalização e sentimentos de realização profissional incompleta. A burnout pode apresentar-se sob diversos tipos de sintomas, como indisposição, insônia, violência, depressão, fraqueza e dores no corpo, dentre outras ${ }^{4}$.

Seu grande foco de incidência está entre os profissionais que se empenham em suas funçóes e lidam com o cuidado ao próximo, como é o caso dos professores. Esses trabalhadores convivem diariamente com riscos psicossociais que colaboram para sintomas que predispóem ao desgaste mental e físico ${ }^{6}$. Além de terem sobrecargas no âmbito trabalhista, esses profissionais não possuem tempo para suas qualificaçóes, o que compromete sua vida profissional e suas realizaçóes pessoais. Diante disso, fica claro que existem diversos fatores que levam ao estresse profissional e que se eles não forem observados com os devidos cuidados, podem levar à ocorrência da síndrome de burnout?

Este estudo objetivou analisar a ocorrência da síndrome de burnout em professores do município de Buenópolis (MG), verificando associaçóes com as características sociodemográficas, econômicas e laborais desses profissionais.

\section{Materiais e métodos}

Foi realizado um estudo de caráter analítico, corte transversal e análise quantitativa. $\mathrm{O}$ cenário deste estudo foi o município de Buenópolis, localizado a $272 \mathrm{~km}$ da cidade de Belo Horizonte, que tem uma população estimada de 10.589 habitantes $^{8}$ e nível do Índice de Desenvolvimento Humano Municipal (IDHM) de
0,669. Investigou-se a ocorrência da síndrome em uma amostra de 71 professores da rede de ensino público do município, que conta com cinco unidades educacionais do pré-escolar ao ensino médio.

Foram incluídos na pesquisa apenas profissionais empregados há no mínimo seis meses nas instituiçóes de ensino envolvidas e que compareceram às reuniôes agendadas para coleta dos dados. Foram excluídos os profissionais afastados do regime de trabalho no momento da pesquisa e uma pessoa com deficiência visual que não conseguiu ler e responder aos questionários.

Utilizou-se para coleta dos dados o formulário MBI (Maslach Burnout Inventory), publicado no início dos anos 1980 e usado para identificação da burnout. O MBI aborda, em 22 itens, as três dimensóes estabelecidas por Maslach e Jackson para a caracterização da burnout. exaustão emocional, despersonalização e reduzida realização profissional. Os próprios participantes preencheram o questionário, que avalia a forma com que o indivíduo vivencia o seu ambiente de trabalho. Foi solicitado aos participantes que respondessem aos itens de acordo com a frequência de ocorrência do acontecimento em uma escala que varia de 0 a 6 . As questóes de 1 a 9 avaliaram o nível de exaustáo emocional; de 10 a 17, a realizaçáo profissional; e as demais relacionam-se à despersonalização 9 .

Os pontos de corte utilizados para o diagnóstico de burnout são de 0 a 15 pontos (baixo), de 16 a 25 (médio) e de 26 a 54 (alto) para exaustáo emocional; de 0 a 2 (baixo), de 3 a 8 (médio) e de 9 a 30 (alto) para a despersonalização; e 0 a 33 (baixo), de 34 a 42 (médio) e de 43 a 48 (alto) para a realização profissional. O diagnóstico de burnout é feito quando fica constatado, a partir do MBI, que o indivíduo tem alta pontuação do nível de cansaço emocional ou despersonalizaçấo, ou baixa do nível de realização pessoal ${ }^{10}$.

Como pode ser visto na Tabela 1 , foi utilizado um formulário socioeconômico com informaçōes sobre idade, sexo, estado civil, renda familiar e escolaridade. Dados associados ao meio laboral também foram avaliados, como carga horária semanal, sensação de valorização e autonomia no trabalho, satisfação com o trabalho e equipamentos fornecidos, acúmulo de serviços docentes em casa e pensamento de desistência da profissão. Esses questionários são respondidos pelos participantes da pesquisa e foram desenvolvidos pelos próprios autores com objetivo de identificar algumas características relacionadas à ocorrência da síndrome de burnout nessa população.

As perguntas foram respondidas na presença das pesquisadoras para sanar as dúvidas dos participantes, caso houvesse alguma durante o preenchimento. A coleta de dados ocorreu mediante reunióes em locais reservados, previamente agendadas, no período entre agosto e setembro de 2016. 
Todos os dados foram tabulados e analisados no software estatístico Statistical Package for Social Sciences (SPSS ), versão 22.0 para Windows. Foram empregados os testes qui-quadrado e exato de Fisher para avaliação da relação entre a ocorrência da burnout e as variáveis sociodemográficas e laborais investigadas. $\mathrm{O}$ nível de significância considerado foi de $5 \%(\mathrm{p}<0,05)$.

Este projeto foi aprovado pelo Comitê de Ética em Pesquisa das Faculdades Unidas do Norte de Minas (Funorte) conforme registrado no parecer de $n^{\circ} 1655450 / 2016$.

\section{Resultados}

O estudo contou com um total de 71 participantes, como pode ser observado na Tabela 1 , em que as maiores proporçôes foram entre as variáveis sociodemográficas: pessoas entre 40 e 49 anos $(39,4 \%)$, do sexo feminino $(84,5 \%)$, casadas $(67,6 \%)$, com renda familiar de até três salários-mínimos $(83,1 \%)$ e nível superior completo (95,8\%). Três dos participantes não apresentaram nível superior (4,2\%), porém, possuem formação em magistério, que os habilita a lecionar para a educação infantil.

Tabela 1: Distribuição de frequências das variáveis sociodemográficas e laborais e ocorrência de síndrome de burnout na população investigada.

\begin{tabular}{|c|c|c|}
\hline Variáveis & $\mathbf{N}$ & $\%$ \\
\hline \multicolumn{3}{|l|}{ Idade } \\
\hline 20 a 29 anos & 3 & 4,2 \\
\hline 30 a 39 anos & 20 & 28,2 \\
\hline 40 a 49 anos & 28 & 39,4 \\
\hline 50 a 59 anos & 20 & 28,2 \\
\hline \multicolumn{3}{|l|}{ Sexo } \\
\hline Feminino & 60 & 84,5 \\
\hline Masculino & 11 & 15,5 \\
\hline \multicolumn{3}{|l|}{ Estado civil } \\
\hline Casado & 48 & 67,6 \\
\hline Solteiro & 17 & 23,9 \\
\hline Viúvo & 3 & 4,2 \\
\hline União estável & 3 & 4,2 \\
\hline \multicolumn{3}{|l|}{ Renda salarial } \\
\hline De $\mathrm{R} \$ 880,00$ até $\mathrm{R} \$ 2.640,00$ & 59 & 83,1 \\
\hline Mais de $\mathrm{R} \$ 2.640,00$ & 12 & 16,9 \\
\hline \multicolumn{3}{|l|}{ Nível de escolaridade } \\
\hline Nível superior incompleto & 3 & 4,2 \\
\hline Nível superior completo & 68 & 95,8 \\
\hline
\end{tabular}

Tabela 1: Continuação.

\begin{tabular}{|c|c|c|}
\hline Variáveis & $\mathbf{N}$ & $\%$ \\
\hline \multicolumn{3}{|c|}{ Carga horária semanal } \\
\hline Até 30 horas & 54 & 76,1 \\
\hline Mais de 30 horas & 17 & 23,9 \\
\hline \multicolumn{3}{|c|}{ Valorização no trabalho } \\
\hline Pouca & 59 & 83,1 \\
\hline Muita & 12 & 16,9 \\
\hline \multicolumn{3}{|c|}{ Autonomia no trabalho } \\
\hline Pouca & 48 & 67,6 \\
\hline Muita & 22 & 31,0 \\
\hline \multicolumn{3}{|c|}{ Satisfaçáo com equipamento de trabalho } \\
\hline Não & 53 & 74,6 \\
\hline Sim & 17 & 23,9 \\
\hline \multicolumn{3}{|c|}{ Leva serviço para casa } \\
\hline Poucas vezes & 28 & 39,4 \\
\hline Muitas vezes & 43 & 60,6 \\
\hline \multicolumn{3}{|l|}{ Gosta do trabalho } \\
\hline Não & 6 & 8,5 \\
\hline Sim & 65 & 91,5 \\
\hline \multicolumn{3}{|c|}{ Já pensou em desistir da profissáo } \\
\hline Não & 35 & 49,3 \\
\hline Sim & 36 & 50,7 \\
\hline \multicolumn{3}{|c|}{ Síndrome de burnout } \\
\hline Ausente & 34 & 47,9 \\
\hline Presente & 37 & 52,1 \\
\hline
\end{tabular}

Fonte: Dados da pesquisa.

No grupo das variáveis laborais, observou-se maior frequência de docentes que relataram cumprir carga horária de até 30 horas semanais $(76,1 \%)$, ter sensação de pouca valorização $(83,1 \%)$ e pouca autonomia na realização do trabalho $(67,6 \%)$, insatisfação com equipamentos disponíveis $(74,6 \%)$, acúmulo de tarefas em domicílio $(60,6 \%)$ e satisfação com o emprego $(91,5 \%)$. Cerca de metade dos participantes informou já ter pensado em desistir da profissão $(50,7 \%)$. Uma pessoa não disponibilizou informaçóes sobre autonomia e satisfação com equipamento de trabalho.

Após análise do MBI, evidencia-se que 52,1\% dos participantes apresentam a síndrome de burnout, conforme a Tabela 1.

$\mathrm{Na}$ Tabela 2, observam-se os resultados da análise estatística bivariada em que se observa maior ocorrência significativa de burnout em indivíduos que se sentem pouco valorizados $(\mathrm{p}=0,001)$, com pouca autonomia no trabalho $(\mathrm{p}<0,001)$, que não gostam do trabalho $(\mathrm{p}=0,014) \mathrm{e}$ que já pensaram em desistir da profissão $(\mathrm{p}<0,001)$. 
Tabela 2: Associações das variáveis sociodemográficas com a ocorrência de síndrome de burnout.

\begin{tabular}{|c|c|c|c|}
\hline Variáveis & Ausente (\%) & Presente (\%) & Valor $\mathbf{p}$ \\
\hline \multicolumn{4}{|l|}{ Idade } \\
\hline 20 a 29 anos & $3(100 \%)$ & $0(0,0 \%)$ & \multirow{4}{*}{0,331} \\
\hline 30 a 39 anos & $9(45,0 \%)$ & $11(55,0 \%)$ & \\
\hline 40 a 49 anos & $13(46,4 \%)$ & $15(53,6 \%)$ & \\
\hline 50 a 59 anos & $9(45,0 \%)$ & $11(55,0 \%)$ & \\
\hline \multicolumn{4}{|l|}{ Sexo } \\
\hline Feminino & $28(46,7 \%)$ & $32(53,3 \%)$ & \multirow[t]{2}{*}{0,631} \\
\hline Masculino & $6(54,5 \%)$ & $5(45,5 \%)$ & \\
\hline \multicolumn{4}{|l|}{ Estado civil } \\
\hline Casado & $23(47,9 \%)$ & $25(52,1 \%)$ & \multirow[t]{2}{*}{0,994} \\
\hline Não casado & $11(47,8 \%)$ & $12(52,2 \%)$ & \\
\hline \multicolumn{4}{|l|}{ Renda } \\
\hline De $\mathrm{R} \$ 880,00$ até $\mathrm{R} \$ 2.640,00$ & $29(49,2 \%)$ & $30(50,38 \%)$ & \multirow[t]{2}{*}{0,636} \\
\hline Mais de $\mathrm{R} \$ 2.640,00$ & $5(41,7 \%)$ & $7(58,3 \%)$ & \\
\hline \multicolumn{4}{|l|}{ Nível de escolaridade } \\
\hline Superior completo & $31(45,6 \%)$ & $37(54,4 \%)$ & \multirow[t]{2}{*}{0,065} \\
\hline Superior incompleto & $3(100 \%)$ & $0(0,0 \%)$ & \\
\hline \multicolumn{4}{|l|}{ Carga horária semanal } \\
\hline Até 30 horas & $27(50,0 \%)$ & $27(50,0 \%)$ & \multirow[t]{2}{*}{0,525} \\
\hline Mais de 30 horas & $7(41,2 \%)$ & $10(58,8 \%)$ & \\
\hline \multicolumn{4}{|l|}{ Valorização no trabalho } \\
\hline Pouca & $23(39,0 \%)$ & $36(61,0 \%)$ & \multirow[t]{2}{*}{$0,001^{*}$} \\
\hline Muita & $11(91,7 \%)$ & $1(8,3 \%)$ & \\
\hline \multicolumn{4}{|l|}{ Autonomia no trabalho } \\
\hline Pouca & $14(29,2 \%)$ & $34(70,8 \%)$ & \multirow[t]{2}{*}{$<0,001^{*}$} \\
\hline Muita & $19(86,4 \%)$ & $3(13,6 \%)$ & \\
\hline \multicolumn{4}{|c|}{ Satisfaçáo com equipamento de trabalho } \\
\hline Náo & $22(41,5 \%)$ & $31(58,5 \%)$ & \multirow[t]{2}{*}{0,095} \\
\hline Sim & $11(64,7 \%)$ & $6(35,3 \%)$ & \\
\hline \multicolumn{4}{|l|}{ Leva serviço para casa } \\
\hline Poucas vezes & $17(60,7 \%)$ & $11(39,3 \%)$ & \multirow[t]{2}{*}{0,081} \\
\hline Muitas vezes & $17(39,5 \%)$ & $26(60,5 \%)$ & \\
\hline \multicolumn{4}{|l|}{ Gosta do trabalho } \\
\hline $\operatorname{Sim}$ & $34(52,3 \%)$ & $31(47,7 \%)$ & \multirow[t]{2}{*}{$0,014^{*}$} \\
\hline Não & $0(0,0 \%)$ & $6(100 \%)$ & \\
\hline \multicolumn{4}{|c|}{ Já pensou em desistir da profissáo } \\
\hline Não & $25(71,4 \%)$ & $10(28,6 \%)$ & \multirow[t]{2}{*}{$<0,001^{*}$} \\
\hline Sim & $9(25,0 \%)$ & $27(75,0 \%)$ & \\
\hline
\end{tabular}

*Valor estatisticamente significativo $(\mathrm{p}<0.05)$.

Os valores foram calculados pelos testes qui-quadrado e exato de Fisher.

Fonte: Dados da pesquisa. 


\section{Discussão}

Os resultados mostram uma proporção substancial de professores que apresentam a síndrome de burnout e uma proporção significativamente maior entre indivíduos que se sentem pouco valorizados e com pouca autonomia na profissão, educadores que declararam não gostar do trabalho e que já pensaram em desistir da docência. Os demais fatores investigados neste estudo não evidenciaram relação estatística com a ocorrência de burnout.

Nesta pesquisa, os professores das escolas públicas do município de Buenópolis apresentaram ocorrência da burnout em 52,1\%; valor relativamente alto, considerando ainda que os dados foram colhidos no início do segundo semestre de 2016, logo após o período de recesso escolar. $\mathrm{O}$ trabalho docente é uma profissão que pode gerar grande estresse, o que faz que boa parte dos professores possa apresentar sinais relacionados à síndrome de burnout. A porcentagem alta verificada neste trabalho pode estar associada à falta de reconhecimento profissional, ocorrência de salários defasados e grande quantidade de alunos indisciplinados, desinteressados pelo processo ensino-aprendizagem. O professor pode se sentir desvalorizado e sem autonomia em sala de aula, pois está trabalhando com estudantes indisciplinados que podem apresentar condutas agressivas, além de lidar com a inexistência de legislação específica que os ampare em tais situaçóes de risco ${ }^{11}$.

O sistema educacional pode levar ao esgotamento profissional pela indisciplina e falta de interesse dos alunos, pela inadequada infraestrutura das escolas e pelos salários baixos, entre outros fatores ${ }^{12}$, o que leva o profissional educador a dar o melhor de si e muitas vezes não conseguir ajudar. Eles passam, então, a apresentar um quadro de desmotivaçáo, demonstrando sinais que levam à geração de doenças. O docente perde seu respeito e agrega medo pela violência, levando-o ao desgosto pela profissão e desistência da vontade de lecionar. Consequentemente, esse panorama leva à perda de bons trabalhadores no sistema educacional, que atualmente representa a última alternativa de escolha profissional para os jovens.

Outras pesquisas investigaram burnout na educação. Um estudo realizado com educadores do ensino pré-escolar $^{13}$ apresentou sinais da síndrome em metade dos participantes, enquanto em outro estudo realizado ${ }^{14}$ com profissionais da educação, $36,8 \%$ demonstram sinais preocupantes para a burnout. Outra pesquisa realizada ${ }^{15}$ com profissionais da educação que trabalham com pessoas com deficiência destacou que $70 \%$ desses profissionais apresentam altos níveis de burnout. E uma pesquisa realizada ${ }^{16}$ com professores de ensino superior apresentou $10,8 \%$ dos participantes com a síndrome e $67,5 \%$ em desenvolvimento, num estado limítrofe.
Por este se tratar de um estudo pontual, realizado apenas no segundo semestre do ano letivo, consideram-se elevados os índices da síndrome de burnout entre os educadores. Ressalta-se, no entanto, a importância da realização de mais estudos de desenho longitudinal para acompanhamento dos professores ao longo do ano que permitam analisar mais fatores relacionados à síndrome, bem como sua ocorrência ao longo do período letivo. Os resultados deste estudo, por exemplo, podem ainda estar subestimados, considerando que algumas pessoas que apresentam as características do burnout possivelmente não teriam disposição e paciência para responder aos formulários ${ }^{17} \mathrm{e}$ poderiam estar afastadas no momento da coleta de dados.

Com a análise dos resultados do estudo, evidencia-se a necessidade de avaliar fatores relacionados ao local de trabalho dos professores, a fim de que se possa sugerir a implantação de propostas de melhorias com mudanças no ambiente organizacional na escola ${ }^{18}$, para que, enfim, os docentes tenham mais autonomia e voz ativa na realizaçáo de tarefas, com sugestóes de pontos a serem melhorados no meio escolar, com o objetivo de que haja prevenção da geração de estresse e desgaste profissional. É preciso também levar aos profissionais o conhecimento da doença por meio de palestras informativas, com a intenção de evitar que a prevalência da síndrome de burnout aumente.

A investigaçáo da burnout dentro do sistema educacional é de extrema importância, pois se não for descoberta e tratada de forma adequada, pode evoluir com mais agravos à saúde dos professores ao longo do tempo. Resultados tâo altos relacionados a este estudo mostram que a educação pode ter perda de qualidade, visto que essa síndrome não afeta apenas o professor, mas todo o sistema educacional, interferindo no desempenho das atividades docentes e prejudicando o processo de ensino-aprendizagem.

O acompanhamento psicopedagógico nas escolas levaria maior apoio aos professores para auxiliar a melhor comunicação entre direção, docentes e alunos, favorecendo assim o clima de confiança e conforto dentro do ambiente escolar ${ }^{19}$. O psicopedagogo ajudaria na identificação de problemas e proposição de estratégias para enfrentamento dessas adversidades com os professores. Esse processo auxiliaria a convivência com os alunos e proporcionaria um vínculo entre a escola e os familiares a partir da abordagem de fatos ocorridos no ambiente escolar, favorecendo todos positivamente.

O esgotamento ocupacional pode levar a um grande número de licenças tiradas anualmente, afastamentos por doença, falta ao trabalho e baixa produtividade em sala, fazendo que seja necessário buscar professores substitutos para ocupar as vagas nesses períodos ${ }^{20}$. Assim acaba-se por aumentar os gastos com contrataçáo - recursos que poderiam ser investidos em um auxílio psicológico constante de caráter preventivo. 


\section{Conclusão}

Este trabalho evidenciou que mais da metade dos professores apresentou a síndrome de burnout. Houve relação estatisticamente significativa na associação de ocorrência da síndrome em indivíduos que se sentem pouco valorizados, com pouca autonomia no trabalho, que não gostam do trabalho e que já pensaram em desistir da profissão.

\section{Referências}

1. França SPS, De Martino MMF, Aniceto EVS, Silva LL. Preditores da síndrome de burnout em enfermeiros de serviços de urgência pré-hospitalar. Acta Paul Enferm. 2012;25(1):68-73.

2. Souza MC, Guimarães ACA, Araújo, CCR. Estresse no trabalho em professores universitários. Rev Bras Ciênc Saúde. 2013;35:1-8.

3. Ferrari R, França FM, Magalhães J. Avaliação da síndrome de burnout em profissionais de saúde: uma revisão integrativa da literatura. Rev Eletr Gestão Saúde. 2012;3(3):1150-65.

4. Abreu SA, Moreira EA, Leite SF, Teixeira CC, Silva ME, Cangussu LMB, et al. Determinação dos sinais e sintomas da síndrome de burnout através dos profissionais da saúde da Santa Casa de Caridade de Alfenas Nossa Senhora do Perpétuo Socorro. Rev. Univ. Vale Rio Verde (Online). 2015;13(1):201-38.

5. Foresto DS, Souza JLE. Síndrome de burnout: indicadores em enfermeiros da atenção primária. Rev Funec Cien Multidisciplinar. 2014;3(5):110-21

6. Carrilo-Esper R, Gómez HK, Estrada IEM. Síndrome de burnout en la práctica medica. Med Int Mex. 2012;28(6):579-84.

7. Andrade PS, Cardoso TAO. Prazer e dor na docência: revisão bibliográfica sobre a síndrome de burnout. Saúde Soc. 2012;21(1):129-40.

8. Instituto Brasileiro de Geografia e Estatística. IBGE Cidades: Buenópolis. 2016. [citado em 2016 mar 18]. Disponível em: http://cod.ibge.gov.br/940
9. Cracco CLAC, Salvador JA. Identificação da síndrome de burnout na equipe de enfermagem de uma unidade de pronto atendimento [monografia]. Lins: Centro Universitário Católico Salesiano Auxilium; 2010.

10. Grunfeld E, Whelan TJ, Zitzelsberger L, Willan AR, Montesanto B, Evans WK. Cancer care workers in Ontario: prevalence of burnout, job stress and job satisfaction. CMAJ. 2000; 163(2):166-9.

11. Zille LP, Cremonezi AM. Estresse no trabalho: um estudo com professores da rede pública estadual de Minas Gerais. Reuna. 2013;18(4):111-28.

12. Machado VR, Boechat IT, Santos, MFR. Síndrome de burnout uma reflexão sobre a saúde mental do educador. Transformar. 2015;7:257-72.

13. Hozo ER, Sucic G, Zaja I. Burnout syndrome among educators in pre-school institutions. Mater sociomed. 2015;27(6):399-403.

14. Sousa JRS, Oliveira GF, Damasceno MMS, Silva ACO. Prevalência da síndrome de burnout em profissionais da educação. Cad Cult Cienc. 2012;11(1):70-9.

15. Ruiz-Calzado I, Llorent VJ. El burnout en los profesionales de la educación que trabajan con personas con discapacidad en Córdoba (España). Influencia de los factores laborales. Ciênci Saúde Coletiva. 2016;21(10):3287-95.

16. Cotrim OS, Wagner LC. Prevalência da síndrome de burnout em professores de uma instituição de ensino superior. Ciênc Mov (Impr.) 2012;14(28):61-70.

17. Benevides-Pereira AMT. Consideraçôes sobre a síndrome de burnout e seu impacto no ensino. Bol psicol. 2012;62(137):155-68.

18. Morte SVRB, Deps VL. Prevenção e tratamento do estresse e da síndrome de burnout em professores da rede pública de ensino. Linkscienceplace. 2015;2(1):62-75.

19. Pontes IAM. Atuação psicopedagógica no contexto escolar: manipulação, não; contribuição, sim. Rev Psicopedag. 2010;27(84):417-27.

20. Mota RVS, Freire Fa LG, Poletto L. Psicopedagogia: a prática do psicopedagogo no ambiente escolar. UC - UNICAMPS CIÊNCIA. 2016;6(6):67-81.

Como citar este artigo:

Araújo VA, Freire JM, Oliveira MVM. Síndrome de burnout em professores das escolas públicas do município de Buenópolis, MG. Rev. Aten. Saúde. 2017;15(52):5-10. 